\title{
Adaptive Cuckoo Search Algorithm for Short-Term Fixed-Head Hydrothermal Scheduling Problem with Reservoir Volume Constraints
}

\author{
Bach Hoang Dinh ${ }^{1 *}$, Thang Trung Nguyen ${ }^{1}$, Dieu Ngoc Vo ${ }^{2}$ \\ ${ }^{1}$ Faculty of Electrical and Electronics Engineering, Ton Duc Thang University, \\ Vietnam, No. 19 Nguyen Huu Tho street, Tan Phong Ward, Dist. 7, Ho Chi Minh \\ City, Vietnam \\ ${ }^{2}$ Department of Power Systems, HCM City University of Technology \\ 268 Ly Thuong Kiet str., 10th dist., Ho Chi Minh City, Viet Nam \\ dinhhoangbach@tdt.edu.vn,nguyentrungthang@tdt.edu.vn,vndieu@gmail.com
}

\begin{abstract}
This paper present three versions of Cuckoo Search Algorithm (CSA) including conventional Cuckoo Search Algorithm (CSA), modified CSA (MCSA) and adaptive CSA (ACSA) for solving the fixed head short-term hydrothermal scheduling (ST-HTS) problem where the reservoir volume constraints and nonconvex fuel cost function of thermal unit as well as the power losses in transmission line are taken into account. Among the applied methods, ACSA is first developed in the study by performing two modifications on second new solution generation via the action of an alien egg to be abandoned. In the ACSA, all initial solutions or all solutions at the end of the previous iteration are evaluated and sorted into two kinds of solution, good solutions with lower fitness function and bad solutions with higher fitness function. The implementation of the first new solution generation first via Lévy flights in the ACSA is carried out similarly to that in MCSA. However, at the second new solution generation the ACSA evaluates the current solutions to choose the best one and use the information of the best one with a random solution to generate the second new solutions via the action of an alien egg to be abandoned. In addition, the probability of an alien egg discovery is considered an adaptive variable, which is set to the largest value at the beginning and decreased as the iteration is increased. Due to the adaptive value of the parameter, the ACSA can search an optimal solution but the trial runs are significantly decreased compared to CSA and MCSA. The performance of the ACSA is validated by testing on two systems and comparing with CSA, MCSA and other existing methods available in the paper.
\end{abstract}

Keywords: Adaptive Cuckoo search algorithm, reservoir volume constraint, fixed head, hydrothermal scheduling

\section{Introduction}

A power system mainly comprises hydropower and thermal plants to supply electricity to load. The primary fuels used at thermal plants are very expensive and become exhausted in the future whereas the water discharged via hydro turbine is considered free and plentiful. Therefore, optimal operation of the system is to decrease the total generation fuel cost of thermal units as much as possible but all hydraulic constraint such as water discharge limits, volume reservoir limits, continuity water and generation limits, and thermal plants constraints including generation limits must be exactly met [1]. These 
methods described above are mainly classified into two groups including deterministic algorithm group and meta-heuristic algorithm group. The former is simply composed of Gradient search [1] and Newton-Raphson method whereas the latter is a large set of meta-heuristic algorithms consisting of simulated annealing approach (SA) [2], evolutionary programming (EP) [4-8], Genetic algorithm (GA) [9], PSO [10-14], improved Bacterial Foraging algorithm (IBFA) [15], Clonal selection algorithm [16]. Among the methods, GS is considered the weakest one since it has been only applied to the problem where conventional hydro generation models were represented as piecewise linear or polynomial approximation, which may be too rough and seems impractical [4]. The Newton-Raphson seems to be more effective than the GS when applied to systems where approximation of the hydro generation can not be performed; however, the method fully depends on the Jacobi matrix which has the dimension depending on the scale of the considered system. This is a disadvantage of the method when applied to a practical system considering many constraints and plants. On the contrary to the deterministic ones, methods in the latter have been successfully and widely applied for solving the short-term hydrothermal scheduling problem taking the reservoir volume constraint into account. Although the SA and GA can tackle the drawbacks that the two deterministic ones must suffer from, the SA has not been widely applied because it spends long simulation time as well as obtaining lower quality solution. Compared to SA and GA, PSO and EP are more effective and robust and have been constantly applied as well as improved to obtain better quality solution and speed up the convergence. In EP [4-5], at creation of offspring step, only Gauss random variable is used to generate offspring and the scaling factor is regarded as a constant whereas in the improved versions of EP $[6,8]$ the number of offspring are generated from randomly generated initial parents using Gauss or Cauchy mutations in addition to using the scaling factor as a variable during the search process. The improvement of EP can improve the solution and speed up convergence and they are superior to conventional EP via only one test system. The application of improved PSO has been presented in [12-13] when a weight factor and a constriction factor were suggested to update new velocity and new position. The weight factor is adaptive meanwhile the constriction factor is fixed at a value depending on the selection of two acceleration constants. The improvement also leads to an optimal solution with shorter execution time but the two studies have reported invalid optimal solution since the water discharge violates the lower limit. In the FIPSO [14], the new version of updated velocity is proposed to obtain better position for each particle and the method has been tested on a system and compared to other methods' result. Despite the statement that the method is superior to other methods, the method is still not recommended to be a strong tool to search optimal solution for the problem since the optimal solution reported in the study is an invalid one violating the lower limit. Farhat et al in study [15] have proposed IBFA for solving the problem; however, the study has shown an invalid optimal solution when they have used more water than availability. Clonal selection algorithm is also a member of meta-heuristic algorithms where the search process is done by performing five steps including initialization, affinity evaluation, Clonal proliferation, mutation and selection. The search process is more complicated than that from evolutionary algorithms like GA, EP and Differential evolution (DE). The method has obtained solution as good as several improved versions of EP.

The original version of Cuckoo search algorithm (CSA) has been developed by Yang and Deb [17]. The CSA is a powerful optimal solution search algorithm once it includes two new solution generations via Lévy flights and the action of an alien egg discovery at each iteration in addition to few easily selected control parameters. In spite of the advantages, Walton et al. [18] have indicated some weak points of the CSA and have built Modified Cuckoo Search Algorithm by carrying out several modifications only on 
the first new solution generation of the CSA and retaining the second new solution generation via the action of an alien egg discovery. The authors have evaluated all solutions and sorted them into two groups, good quality group and bad quality group based on their fitness function value. Each solution in the two groups is then newly generated via Lévy flights by different ways corresponding to its feature.

In this paper, an adaptive cuckoo search algorithm (ACSA) is first developed for solving the ST-HTS problem by using two modifications from MCSA on the first new solution generation via Lévy flights and performing two other modifications on the second new solution generation via the action of an alien egg discovery.

In the ACSA, all new solutions of the two groups are combined into only one group and the evaluation of the current solutions is done to determine the best one which is used to exchange information with a random solution to produce new solution for the second time. On the other hand, the probability of a fraction of eggs to be abandoned, which is fixed at a constant in CSA and MCSA, is adaptive in the ACSA. The first modification aim to search a better optimal solution meanwhile the second modification is focus on the reduction of the trial runs.

The performance of the proposed ACSA method has been tested on two systems consisting of the first system with small scale and quadratic fuel cost function of thermal unit and the second system with large scale and nonconvex fuel cost function of thermal units. The obtained results in terms of solution quality and execution time from the proposed ACSA are compared to methods available in the paper including CSA and MCSA.

\section{Problem Formulation}

The hydrothermal system consisting of $N_{l}$ thermal units and $N_{2}$ hydro units scheduled in $M$ time sub-intervals with $t_{m}$ hours for each is mathematically formulated. The problem aims to minimize total cost of thermal units subject to the system and unit constraints.

The objective function of the problem is as follows:

$$
F=\sum_{m=1}^{M} \sum_{i=1}^{N_{1}} t_{m}\left[\begin{array}{l}
a_{s i}+b_{s i} P_{s i, m}+c_{s} P_{s i, m}^{2} \\
+\left|d_{s i} \times \sin \left(e_{s i} \times\left(P_{s i}^{\min }-P_{s i, m}\right)\right)\right|
\end{array}\right]
$$

where $a_{s i}, b_{s i}, c_{s i}, d_{s i}, e_{s i}$ are fuel cost coefficients of thermal plant $i$.

subject to

1) Power load demand balance: The total power generation from thermal and hydro units must satisfy the load demand plus power losses in transmission lines:

$$
\sum_{i=1}^{N_{1}} P_{s i, m}+\sum_{j=1}^{N_{2}} P_{h j, m}-P_{L, m}-P_{D, m}=0
$$

where the power losses in transmission lines are calculated using Kron's formula as follows:

$$
P_{L, m}=\sum_{i=1}^{N_{1}+N_{2}} \sum_{j=1}^{N_{1}+N_{2}} P_{i m} B_{i j} P_{j m}+\sum_{i=1}^{N_{1}+N_{2}} B_{0 i} P_{i m}+B_{00}
$$


where $P_{D, m}$ and $P_{L, m}$ are total system load demand and power loss in the transmission line at subinterval $m$.

2) The total water discharge constraint: The available total water for all hydro units is determined by:

$$
Q_{j, m}=t_{m} q_{j, m}
$$

where $q_{j, m}$ is water discharge rate obtained by

$$
q_{j, m}=a_{h j}+b_{h j} P_{h j, m}+c_{h j} P_{h j, m}^{2}
$$

3) The continuity of reservoir volume: The water continuity of hydro power plants is represented by:

$$
V_{j, m-1}-V_{j, m}+I_{j, m}-Q_{j, m}-S_{j, m}=0
$$

where $V_{j, m}, I_{j, m}$ and $S_{j, m}$ are reservoir volume, water inflow and spillage discharge rate of $j^{\text {th }}$ hydropower plant in $m^{\text {th }}$ interval, respectively.

4) Initial and final reservoir storage: The initial and final reservoir volumes are predetermined:

$$
V_{j, 0}=V_{j, \text { initial }} ; \quad V_{j, M}=V_{j, \text { End }}
$$

5) Reservoir storage limits: The reservoir volume of hydro power plants is limited by their upper and lower boundaries:

$$
V_{j, \min } \leq V_{j, m} \leq V_{j, \max } ; j=1,2, \ldots, N_{2} ; m=1,2, \ldots, M
$$

where $V_{j, \max }$ and $V_{j, \min }$ are the maximum and minimum reservoir storage of the hydro plant $j$, respectively.

6) Water discharge rate limits: The water discharge rate of hydro power plants is limited by their upper and lower boundaries:

$$
q_{j, \min } \leq q_{j, m} \leq q_{j, \max } ; j=1,2, \ldots, N_{2} ; m=1,2, \ldots, M
$$

where $q_{j, \text { max }}$ and $q_{j, \min }$ are the maximum and minimum water discharge of the hydro plant $j$.

7) Generator operating limits: The power outputs of thermal and hydro units are limited by their upper and lower boundaries:

$$
\begin{gathered}
P_{s i, \text { min }} \leq P_{s i, m} \leq P_{s i, \text { max }} ; i=1,2, \ldots, N_{1} ; m=1,2, \ldots, M \\
P_{h j, \min } \leq P_{h j, m} \leq P_{h j, \max } ; j=1,2, \ldots, N_{2} ; m=1,2, \ldots, M
\end{gathered}
$$


where $P_{s i \text { max }}, P_{s i, \min }$ and $P_{h j, \max }, P_{h j, \min }$ are maximum, minimum power output of thermal plant $i$ and hydro plant $j$, respectively.

\section{Calculation Of Slack Thermal Unit And Slack Hydro Units}

Suppose that the power output of all thermal units except unit 1 and all reservoir volumes at the end of each scheduled subinterval are known.

The total water discharge over the $t_{m}$ hours is then calculated using (6) as follows:

$$
Q_{j, m}=V_{j, m-1}-V_{j, m}+I_{j, m}-S_{j, m} ; \quad m=1, \ldots, M
$$

The water discharge $q_{j, m}$ is calculated using (3) and then hydro generation $P_{h j, m}$ can be obtained using (5).

Thermal unit 1 is selected as the slack unit. The power output of slack thermal unit 1 is calculated using the power balance equation as follows [20]:

$$
P_{s 1, m}=P_{D, m}+P_{L, m}-\sum_{i=2}^{N_{1}} P_{s i, m}-\sum_{j=1}^{N_{2}} P_{h j, m}
$$

\section{Adaptive Cuckoo Search Algorithm}

The Adaptive Cuckoo search algorithm is proposed in the paper to improve the performance of the MCSA, which is first proposed by Walton et al in [18].

In the ACSA, the first modification is to determine an increased value to update the new solution via the discovery of alien eggs. All the eggs in the good quality and bad quality groups after fulfilling the first new solution generation via Lévy flights are put in the integrated group and evaluated to choose the best one. The obtained best egg will be employed to obtain the increased value. The new solution is obtained by the following equation.

$$
X_{d}^{\text {new }}= \begin{cases}X_{d}+\operatorname{rand}\left[\operatorname{rand}_{p}(X)-X_{G b e s t}\right] & \text { if rand }<P a \\ X_{d} & \text { otherwise }\end{cases}
$$

where the probability of $\mathrm{Pa}$ is adaptive as the following equation:

$$
P_{a}=P_{a, \max }-\frac{G}{G_{\max }}\left(P_{a, \max }-P_{a, \min }\right)
$$

In contrast to CSA and MCSA, the parameter is a variable in stead of a predetermined constant in range from 0 to 1 .

As observed from [19-21], the optimal solutions were obtained at a value of $\mathrm{Pa}$ in range of 0.1-0.5 or 0.5-0.9. Therefore, the value of $P_{a, \max }$ and $P_{a, \min }$ in the ACSA are respectively set to 0.9 and $0.5,0.5$ and 0.1 , and 0.9 and 0.1 .

The adaptive probability is the second modification of the ACSA on the MCSA.

\section{Implementation of the Proposed Adaptive Cuckoo Search Algorithm For ST-HTS Problem}

The proposed ACSA method is performed for solving the ST-HTS problem considering the reservoir volume constraints as follows: 


\subsection{Initialization}

In the ACSA, each host nest $X_{d}=\left[\begin{array}{lll}P_{s i, m, d} & V_{j, m, d}\end{array}\right]$ represents a solution containing variables in which $d=1, \ldots, N_{p}, N_{P}$ is the number of nests, $P_{s i, m, d}$ and $V_{j, m, d}$ are power output of the $i^{\text {th }}$ thermal unit and reservoir volume of the $j^{\text {th }}$ hydropower plant at the $m^{\text {th }}$ subinterval corresponding to the $d^{\text {th }}$ nest among the fixed nests, respectively.

Each element in nest $d$ of the population is randomly initialized as follows:

$$
\begin{array}{r}
P_{s i, m, d}=P_{s i, \min }+\operatorname{rand}^{*}\left(P_{s i, \max }-P_{s i, \min }\right) ; \\
i=2, \ldots, N_{1} ; m=1, \ldots, M \\
V_{j, m, d}=V_{j, \min }+\operatorname{rand}^{*}\left(V_{j, \max }-V_{j, \text { min }}\right) ; \\
j=1, \ldots, N_{2} ; m=1, \ldots, M-1
\end{array}
$$

where rand is a random number ranging in $[0,1]$.

Certainly, the upper and lower limits of each nest are respectively $X_{\min }=\left[\begin{array}{ll}P_{s i, \min } & V_{j, \min }\end{array}\right]$ and $X_{\max }=\left[P_{s i, \max } V_{j, \max }\right]$.

The power output of all hydro units and slack thermal unit 1 are obtained as described in section III.

The fitness function consisting of objective function and penalty terms and representing the quality of a solution is determined as follows.

$$
F T_{d}=\left(\begin{array}{l}
\sum_{m=1}^{M} \sum_{i=1}^{N 1} F_{i}\left(P_{s i, m, d}\right)+K_{s} \sum_{m=1}^{M}\left(P_{s 1, m, d}-P_{s 1}^{\mathrm{lim}}\right)^{2} \\
+K_{q} \sum_{j=1}^{N 2} \sum_{m=1}^{M}\left(q_{j, m, d}-q_{j}^{\lim }\right)^{2}
\end{array}\right)
$$

where $K_{s}$ and $K_{q}$ are penalty factors; $P_{s l, m, d}$ is the power of the slack thermal unit 1 at the $m^{\text {th }}$ subinterval of the $d^{\text {th }}$ nest.

The limits in (18) are calculated as follows:

$$
\begin{aligned}
& P_{s 1}^{\lim }=\left\{\begin{array}{ll}
P_{s 1, \max } & \text { if } P_{s 1, m, d}>P_{s 1, \max } \\
P_{s 1, \min } & \text { if } P_{s 1, m, d}<P_{s 1, \min } \\
P_{s 1, m, d} & \text { otherwise }
\end{array} ; m=1, \ldots, M\right. \\
& q_{j}^{\lim }=\left\{\begin{array}{lll}
q_{j, \max } & \text { if } q_{j, m, d}>q_{j, \max } \\
q_{j, \min } & \text { if } q_{j, m, d}<q_{j, \min } & ; j=1, . ., N_{2} ; \\
q_{j, m, d} & \text { otherwise } & m=1, . ., M
\end{array}\right.
\end{aligned}
$$

where $P_{s l, \max }$ and $P_{s l, \min }$ are respectively the minimum and maximum powers of the slack thermal unit 1.

All the solutions are first evaluated based on the fitness function values and the solution with the lowest value is called Global best solution Gbest whereas the remaining solutions are called local best solution Xbestd.

\subsection{The First New Solution Generation via Lévy Flights}

The first new solution generation via Lévy flights implemented in the MCSA [21] is applied again in the section. The detail is as below.

All current solutions are ranked in the descending order of fitness value and then 
divided into two groups. The solutions with high fitness function value are put in bad quality group, called $\mathrm{Xbad}_{d}$ and the other ones are put in the good quality group, called Xgood $_{d}$. Two eggs in the good group, which are picked to exchange information and produce increased values, include one intentionally chosen solution in order, $\operatorname{Xgood}_{d}$ and one randomly chosen solution, $\mathrm{Xgood}_{r}$.

\subsubsection{Generation of new solution for the abandoned group}

Based on the modification applied to the bad quality group with $d=N g+1, \ldots, N_{p}$ where $\mathrm{Ng}$ is the number of solutions in the good quality group, the optimal path for the Lévy flights is calculated using Mantegna's algorithm as follows [22]:

$$
\mathrm{Xbad}_{d}^{\text {new }}=\left(\mathrm{Xbad}_{d}+\operatorname{rand} \times \Delta \mathrm{Xbad}_{d} / \sqrt{G}\right)
$$

where $G$ is the number current iteration and $\Delta X_{d}$ is obtained by [21]:

$$
\Delta \text { Xbad }_{d}=\text { Levy } \times\left(\text { Xbad }_{d}-\text { Gbest }_{d}\right) ;
$$

\subsubsection{Generation of new solution for the top egg group}

The modification is applied to the eggs in the top group with $d=1, \ldots, N g$ (where $\mathrm{Ng}$ is the number of solutions in the good quality group). The optimal path for the Lévy flights is calculated using Mantegna's algorithm as follows:

$$
X_{t o p}^{\text {new }}=\text { Xtop }_{d}+\text { rand } \times \Delta \text { Xgood }_{d}
$$

The value $\Delta X \operatorname{good}_{d}$ is determined depending on considered cases as follows:

a) Case 1: The same egg is picked twice

$$
\Delta \operatorname{Xgood}_{d}=\operatorname{Levy} \times\left(\text { Xtop }_{d}-\text { Gbest }\right) / G^{2}
$$

b) Case 2: Both eggs have the same fitness value function

$$
\Delta \operatorname{Xgod}_{d}=\left(\operatorname{Xgood}_{d}-\operatorname{Xgood}_{r}\right) / 2
$$

c) Case 3: The random egg has lower fitness than egg $d$,

$$
\Delta \operatorname{Xgood}_{d}=\left(\operatorname{Xgood}_{r}-\operatorname{Xgood}_{d}\right)(1+\sqrt{5}) / 2
$$

or the random egg has higher fitness than $\operatorname{egg} d$,

$$
\Delta \operatorname{Sood}_{d}=\left(\operatorname{Xgood}_{d}-\operatorname{Xgood}_{r}\right)(1+\sqrt{5}) / 2
$$

There are no criteria to ensure that the new solutions always satisfy their bounds. Therefore, they are checked and set to upper limit or lower limit if they are higher than upper limits or less than lower limit.

The power output of $N_{2}$ hydro units and the slack thermal unit are then obtained as 
shown in section III. The fitness value is calculated using equations (18). The nest corresponding to the best fitness function is then set to the best nest Gbest.

\subsection{The second new solution generation via discovery of alien eggs}

In the second new solution generation, the two modification described in section $\mathrm{V}$ is applied. The new solutions are then checked and redefined if they violate limits.

\subsection{Terminating criteria}

The whole search process of ACSA is terminated as the maximum number of iteration is reached.

\subsection{Overall Procedure}

The overall procedure of the proposed ACSA for solving the ST- HTS problem is described as follows.

Step 1: Set three control parameters consisting of number of host nests $N_{p}$, maximum and minimum probability, $P_{a, \max }$ and $P_{a, \min }$, and maximum number of iterations $N_{\max }$.

Step 2: Initialize a population of $N_{p}$ host nests and calculate the power output of all hydro units and the slack thermal unit. Set the initial iteration counter $G=1$.

Step 3: Evaluate quality of solutions using (18) and classify the solutions into bad quality group $\mathrm{Xbad}_{d}$ and good quality group, $\operatorname{XTop}_{d}(\mathrm{~d}=1, \ldots$, Notop $)$. The best one in good group is called Gbest.

Step 4: Generate new solutions via Lévy flights described and calculate the power output of all hydro units and the slack thermal unit.

Step 5: Compare the each old and each new solution at the same nest to keep better one which obtains lower fitness function value.

Step 6: Combine the kept solutions in good quality group and bad quality group and evaluate quality to determine the best egg with the lowest fitness function.

Step 7: Generate new solutions via the discovery of alien eggs and calculate the power output of all hydro units and the slack thermal unit.

Step 8: Calculate (18) to compare the each old and each new solution at the same nest to keep better one which obtains lower fitness function value.

Step 9: Determine the best solution, Gbest.

Step 10: If $G<G_{\max }, G=G+1$ and go back to Step 3. Otherwise, terminate the iterative procedure.

\section{Numerical Results and Discussion}

The performance of the proposed ACSA is validated by testing on two test systems in which the first one has one thermal plant with quadratic cost function and one hydro plant and another consists of four thermal plants with nonconvex fuel cost function and four hydro plants [1]. The two systems are scheduled in 3 days with six 12-hour intervals. The transmission losses are neglected in the first system and included in the second one. For system 2, the data of thermal units is taken from table A8 in [20], data of 4 hydro units is from system 1 and transmission loss coefficients is given in Appendix. The ACSA is coded in Matlab platform and run fifty independent trials for each case on a $1.8 \mathrm{GHz}$ PC with $4 \mathrm{~GB}$ of RAM.

For comparison, both the CSA and the MCSA are also implemented for solving the two systems. 


\subsection{Selection of parameter for the CSA based methods}

The number of nests and maximum number of iterations for the three methods are indicated in table 1. In addition, the probability of CSA and MCSA is tuned in range from 0.1 to 0.9 with a step of 0.1 whereas the value of $\mathrm{P}_{a \text {,max }}$ and $\mathrm{P}_{\mathrm{a}, \min }$ in ACSA are respectively set to 0.9 and $0.5,0.5$ and 0.1 , and 0.9 and 0.1 . Clearly, the ACSA can reduce 3 times the probability compared to CSA and MCSA. The number of nests and the maximum number of iterations for the two systems are given in Table 1 .

Table 1. Selection of Control Parameters

\begin{tabular}{|l|l|l|l|l|}
\hline \multirow{2}{*}{ Method } & \multicolumn{2}{|l|}{ System 1 } & \multicolumn{2}{l|}{ System 2 } \\
\cline { 2 - 5 } & NP & Gmax & NP & Gmax \\
\hline CSA & 8 & 100 & 50 & 3500 \\
\hline MCSA & 8 & 100 & 36 & 3500 \\
\hline ACSA & 8 & 100 & 36 & 3500 \\
\hline
\end{tabular}

\subsection{System 1 with Convex Cost Function of Thermal Unit}

The obtained results in detail from CSA, MCSA and ACSA are respectively given in Tables 2, 3 and 4. As indicated in the tables, CSA respectively obtains the best minimum, average, maximum and standard deviation costs at $P a=0.6,0.1,0.8$ and 0.1 meanwhile these best cost is obtained at the same value of $P a=0.8$ for MCSA, and the proposed ACSA obtains the best, average and maximum costs at $P_{a, \max }=0.9$ and $P_{a, \min }=$ 0.5 . Obviously, the best cost obtained by ACSA is approximate to that from CSA and MCSA but the standard deviation is much better. The best minimum cost from the three methods is also compared to the best cost obtained by other methods given in Table 5 such as such as Gradient search (GS) [1], Simulated annealing approach (SA) [3], Evolutionary programming (EP) [4-5], Fast EP (FEP), Improved fast EP (IFEP) [6], Running Improved fast EP (RIFEP) [8], clonal selection algorithm [16]. The comparison has revealed that the ACSA can obtain less minimum cost than GS [1], SA [2], EP [3], and EP [4] and approximate that from other methods. Moreover, the ACSA spends shorter simulation time than the most of methods excluding CSA and MCSA for obtaining the best optimal solution. The convergence characteristics of the three CSA methods are shown in Figure 1.

Table 2. Results Obtained by CSA for System 1

\begin{tabular}{|c|l|c|c|c|l|}
\hline $\mathrm{p}_{\mathrm{a}}$ & Min. cost $(\$ / \mathrm{h})$ & $\begin{array}{c}\text { Average } \\
\text { cost }(\$ / \mathrm{h})\end{array}$ & $\begin{array}{c}\text { Max. } \\
\text { cost }(\$ / \mathrm{h})\end{array}$ & $\begin{array}{c}\text { Std. dev. } \\
(\$ / \mathrm{h})\end{array}$ & CPU time $(\mathrm{s})$ \\
\hline 0.1 & 709862.0644 & 709931.303 & 711753.74 & 271.20 & 0.08 \\
\hline 0.2 & 709862.0669 & 710085.314 & 715936.90 & 884.58 & 0.07 \\
\hline 0.3 & 709862.0676 & 709984.017 & 712720.59 & 467.11 & 0.08 \\
\hline 0.4 & 709862.0995 & 710043.040 & 714846.03 & 778.46 & 0.08 \\
\hline 0.5 & 709862.0541 & 710315.478 & 726879.29 & 2437.41 & 0.07 \\
\hline 0.6 & 709862.0494 & 710016.545 & 715532.96 & 809.64 & 0.08 \\
\hline 0.7 & 709862.0544 & 709954.689 & 713911.29 & 568.35 & 0.08 \\
\hline 0.8 & 709862.0503 & 709984.895 & 711726.57 & 376.82 & 0.08 \\
\hline 0.9 & 709862.0661 & 710187.150 & 723528.22 & 1913.95 & 0.07 \\
\hline
\end{tabular}


Table 3. Results Obtained by MCSA for System 1

\begin{tabular}{|c|c|c|c|c|l|}
\hline$p_{a}$ & $\begin{array}{c}\text { Min. cost } \\
(\$ / h)\end{array}$ & $\begin{array}{c}\text { Average } \\
\text { cost }(\$ / h)\end{array}$ & $\begin{array}{c}\text { Max. } \\
\text { cost }(\$ / h)\end{array}$ & $\begin{array}{c}\text { Std. dev. } \\
(\$ / h)\end{array}$ & $\begin{array}{c}C P U \\
\text { time }(s)\end{array}$ \\
\hline 0.1 & 709866.177 & 710458.729 & 715618.483 & 922.553 & 0.14 \\
\hline 0.2 & 709862.919 & 710477.340 & 717426.705 & 1169.337 & 0.13 \\
\hline 0.3 & 709862.524 & 710315.697 & 714193.848 & 833.334 & 0.13 \\
\hline 0.4 & 709862.130 & 710032.901 & 711943.064 & 364.173 & 0.13 \\
\hline 0.5 & 709862.066 & 710137.651 & 715095.996 & 803.715 & 0.14 \\
\hline 0.6 & 709862.079 & 710020.531 & 711382.933 & 386.170 & 0.13 \\
\hline 0.7 & 709862.066 & 709974.313 & 711053.453 & 280.933 & 0.14 \\
\hline 0.8 & 709862.070 & 709978.559 & 711966.777 & 368.597 & 0.13 \\
\hline 0.9 & 709862.054 & 709905.500 & 710966.963 & 189.488 & 0.13 \\
\hline
\end{tabular}

Table 4. Results Obtained by The Proposed ACSA for System 1

\begin{tabular}{|c|c|c|c|c|c|c|}
\hline $\begin{array}{c}\mathrm{p}_{\mathrm{a},} \\
\max \end{array}$ & $\begin{array}{c}\mathrm{p}_{\mathrm{a}}, \\
\min \end{array}$ & $\begin{array}{c}\text { Min. cost } \\
(\$ / \mathrm{h})\end{array}$ & $\begin{array}{c}\text { Average } \\
\text { cost }(\$ / \mathrm{h})\end{array}$ & $\begin{array}{c}\text { Max. cost } \\
(\$ / \mathrm{h})\end{array}$ & $\begin{array}{c}\text { Std. dev. } \\
(\$ / \mathrm{h})\end{array}$ & $\begin{array}{c}\text { CPU } \\
\text { time }(\mathrm{s})\end{array}$ \\
\hline 0.9 & 0.5 & 709862.0490 & 709862.12 & 709862.83 & 0.16 & 0.12 \\
\hline 0.5 & 0.1 & 709862.0502 & 709867.65 & 709989.94 & 18.69 & 0.12 \\
\hline 0.9 & 0.1 & 709862.0530 & 709862.55 & 709868.38 & 1.05 & 0.12 \\
\hline
\end{tabular}

Table 5. Comparison of Result for System 1

\begin{tabular}{|l|l|c|}
\hline \multicolumn{1}{|c|}{ Method } & Fuel cost $(\$)$ & $\begin{array}{c}\text { CPU } \\
\text { time }(\mathrm{s})\end{array}$ \\
\hline GS [1] & 709877.38 & $\mathrm{NA}$ \\
\hline SA [3] & 709874.36 & 901 \\
\hline EP [4] & 709862.06 & 8 \\
\hline EP [5] & 709863.29 & 264 \\
\hline FEP [6] & 709862.05 & 101.4 \\
\hline IFEP [6] & 709862.05 & 59.7 \\
\hline RIFEP [8] & 709862.05 & $\mathrm{NA}$ \\
\hline $\begin{array}{l}\text { Clonal } \\
\text { selection [16] }\end{array}$ & 709862.05 & 4.54 \\
\hline CSA & 709862.0494 & 0.086 \\
\hline MCSA & 709862.054 & 0.13 \\
\hline ACSA & 709862.0490 & 0.12 \\
\hline
\end{tabular}




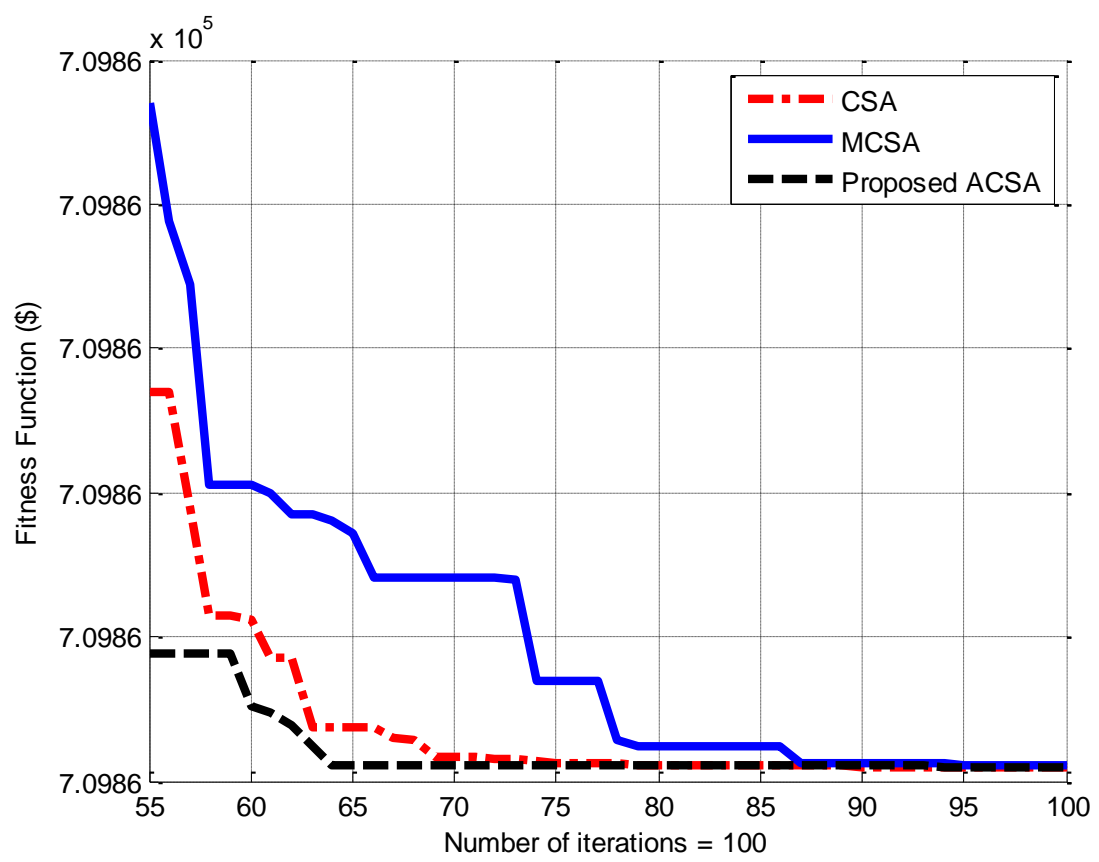

Figure 1. Convergence Characteristics of the Three Methods for System 1

\subsection{System 2 with Nonconvex Cost Function OF Thermal Units}

The best results in terms of minimum cost, average cost, maximum cost, standard deviation cost and execution time obtained by the three CSA methods are compared in Table 6. It is clearly seen from the table that the ACSA is very efficient to search optimal solution since it obtains better minimum cost than CSA and MCSA. Furthermore, the ACSA is faster than CSA. The fitness convergence characteristic comparison indicated in figure 2 shows that the ACSA obtains better solution than CSA at each iteration and worse solution that MCSA when the iteration is less than 1500. As the iteration is higher than 1500, the ACSA reaches better solution than MCSA.

The analysis on the obtained results points out that the ACSA is very efficient for the short-term hydrothermal scheduling problem where the volume constraint of hydro plant and nonconvex fuel cost function of thermal units are taken into consideration.

Table 6. Comparison of Result Obtained from the Three CSA Methods for System 2

\begin{tabular}{|l|l|l|c|c|c|}
\hline Method & $\begin{array}{c}\text { Min. cost } \\
(\$ / \mathrm{h})\end{array}$ & $\begin{array}{c}\text { Average } \\
\text { cost }(\$ / \mathrm{h})\end{array}$ & $\begin{array}{c}\text { Max. } \\
\operatorname{cost}(\$ / \mathrm{h})\end{array}$ & $\begin{array}{c}\text { Std. dev. } \\
(\$ / \mathrm{h})\end{array}$ & $\begin{array}{c}\text { CPU } \\
\text { time }(\mathrm{s})\end{array}$ \\
\hline CSA & 76781.9876 & 77283.19 & 77811.08 & 226.454 & 46.4 \\
\hline MCSA & 76756.79 & 77283.35 & 77965.5 & 212.525 & 35.4 \\
\hline ACSA & 76694.4746 & 77287.866 & 77953.16 & 252.928 & 35.9 \\
\hline
\end{tabular}




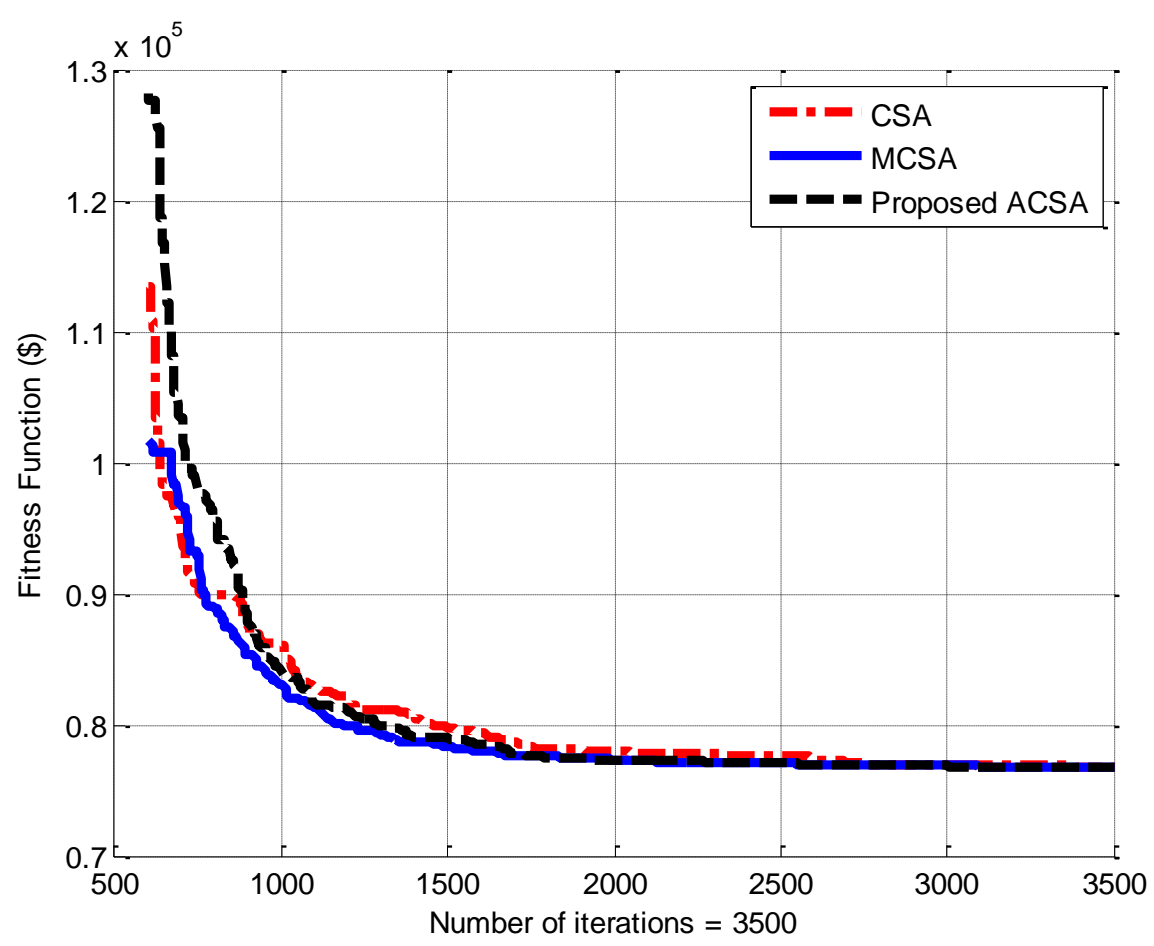

Figure 2. Convergence Characteristics of the Three Methods For System 2

\section{Conclusions}

In this paper, three versions of Cuckoo search algorithm are applied for solving the short-term hydrothermal scheduling problem. Among the methods, adaptive CSA is first developed in the study to improve the performance of the MCSA. To validate the efficiency of ACSA, two different systems are employed where the first system is composed of one hydropower plant and one thermal plant with quadratcand the second one comprises four hydro and four thermal plants with nonconvex fuel cost function. The power losses in transmission line are neglected in system 1 and considered in system 2. The cost comparison and execution time comparison for the system 1 indicates that the ACSA is more effective and robust than other methods including CSA and MCSA meanwhile the comparison for the large system 2 points out that the ACSA is superior to CSA and MCSA since it obtain better solution than the two methods and it is faster than CSA. Consequently, the ACSA is a promising powerful meta-heuristic algorithm for solving short-term hydro thermal scheduling problem considering large scale system and complex constraints as well as non-differential objective function.

\section{References}

[1] A. J. Wood and B. F. Wollenberg, "Power Generation, Operation, and Control", 2nd ed., New York: Wiley, (1996).

[2] M. F. Zaghlool and F. C. Trutt, "Efficient methods for optimal scheduling Of fixed head hydrothermal power systems", IEEE Transactions on Power Systems, vol. 3, issue 1, (1998), pp. 2430.

[3] K. P. Wong, and Y. W. Wong, "Short-term hydrothermal scheduling, part-I: simulated annealing approach”, IEE Proc Part, vol. 141, (1994), pp. 497-501.

[4] P. Yang, H. Yang and C. Huang, "Scheduling short-term hydrothermal generation using evolutionary programming techniques", Generation Transmission and Distribution, IEE Proceedings, (1996), pp. 143 371-376. 
[5] P. K. Hota, R. Chakrabarti, P. K. Chattopadhyay, "Short-term hydrothermal scheduling through evolutionary programming technique", Electric Power Systems Research, vol. 52, (1999), pp. 189 196.

[6] N. Sinha, R. Chakrabarti and P. K. C. Haya, "Fast evolutionary programming techniques for short-term hydrothermal scheduling", Electric Power Syst. Res., vol. 66, (2003), pp. 97-103.

[7] C. Nallasivan, D. S. Suman, J. Henry, S. Ravichandran, "A Novel Approach for Short-Term Hydrothermal Scheduling Using Hybrid Technique", Power India Conference, IEEE, (2006), pp. 1-5.

[8] B. Türkay, F. Mecitoğlu, S. Baran, "Application of a fast evolutionary algorithm to short-term hydrothermal generation scheduling", Energy Sources, Part B: Economics, Planning, and Policy, vol.6, (2011), pp.395-405.

[9] H. C. Chang, P. H. Chen, "Hydrothermal generation scheduling package: A genetic based approach", IEE Proc.-Gener Transm. Distrib., vol. 145, issue. 4, (1998), pp. 451-57.

[10] H. Samudi, P. D. Gautham, C. O. Piyush, T. S. Sreeni amd S. Cherian, "Hydro Thermal Scheduling using Particle Swarm Optimization", IEEE conference in India, (2008), pp. 1-5.

[11] S. Thakur, C. Boonchay and W. Ongsakul, "Optimal Hydrothermal Generation Scheduling using SelfOrganizing Hierarchical PSO”, Power and Energy Society General Meeting, IEEE, (2010), pp. 1-6.

[12] S. Padmini and C. C. A. Rajan, "Improved PSO for Short Term Hydrothermal Scheduling", IEEE conference in India, (2011) pp. 332-334.

[13] S. Padmini, C. C. A. Rajan and P. Murthy, "Application of Improved PSO Technique for Short Term Hydrothermal Generation Scheduling of Power System", Second International Conference, SEMCCO 2011, Visakhapatnam, Andhra Pradesh, India, Proceedings, Part I, (2011), pp. 176-182.

[14] M. S. Fakhar, S. A. R. Kashif and M. A. S. T. Hassan, "Non cascaded short-term hydro-thermal scheduling using fully-informed particle swarm optimization", Electrical Power and Energy Systems, vol. 73, (2015), pp. 983-990.

[15] I. A. Farhat and M. E. E. Hawary, "Short-Term Hydro-Thermal Scheduling Using an Improved Bacterial Foraging Algorithm", Electrical Power \& Energy Conference (EPEC), IEEE, (2009), pp. 1-5.

[16] R. K. Swain, A. K. Barisal, P. K. Hota and R. Chakrabarti, "Short-term hydrothermal scheduling using clonal selection algorithm”, Electrical Power \& Energy Systems, vol.33, (2011), pp. 647-656.

[17] X. S. Yang, S. Deb, "Cuckoo search via Lévy flights", Proceedings of the World Congress on Nature \& Biologically Inspired Computing (NaBIC), India, (2009), pp. 210-214

[18] S. Walton, O. Hassan, K. Morgan and M. R. Brown, "Modified cuckoo search: A new gradient free optimisation algorithm", Chaos, Solutions \& Fractals, vol. 44, (2011), pp. 710-718.

[19] V. N. Dieu, S. Peter and W. Ongsakul, "Cuckoo search algorithm for non-convex economic dispatch," IET Generation, Transmission \& Distribution, vol. 7, (2003), pp. 645-54.

[20] N. T. Thang, V. N. Dieu and T. V. Anh, "Cuckoo search algorithm for short-term hydrothermal scheduling”, Applied Energy, vol. 132, (2014), pp. 276-287.

[21] N. T. Thang, V. N. Dieu, "Modified Cuckoo search algorithm for short-term hydrothermal scheduling", Electrical Power and Energy Systems, vol. 65, (2015), pp. 271-281.

[22] R. N. Mantegna, "Fast, accurate algorithm for numerical simulation of Levy stable stochastic processes", Phys Rev E., vol. 49, (1994), pp. 4677-4683.

\section{APPENDIX}

Transmission loss coefficients for system 2:

$\mathrm{B}=\left[\begin{array}{cccccc}0.000049 & 0.000014 & 0.000015 & 0.000015 & 0.000020 & 0.000017 \\ 0.000014 & 0.000045 & 0.000016 & 0.000020 & 0.000018 & 0.000015 \\ 0.000015 & 0.000016 & 0.000039 & 0.000010 & 0.000012 & 0.000012 \\ 0.000015 & 0.000020 & 0.000010 & 0.000040 & 0.000014 & 0.000010 \\ 0.000020 & 0.000018 & 0.000012 & 0.000014 & 0.000035 & 0.000011 \\ 0.000017 & 0.000015 & 0.000012 & 0.000010 & 0.000011 & 0.000036 \\ 0.000020 & 0.000018 & 0.000012 & 0.000014 & 0.000035 & 0.000011 \\ 0.000017 & 0.000015 & 0.000012 & 0.000010 & 0.000011 & 0.000011\end{array}\right]$




\section{Authors}
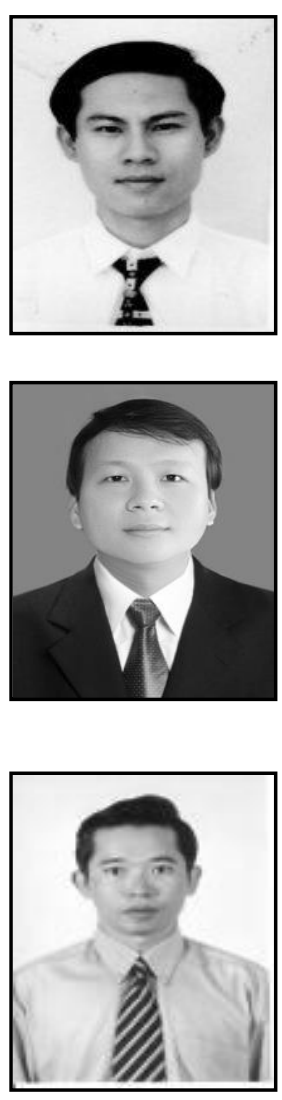

Bach Hoang Dinh, he received B.Eng and M.Eng degrees from the HCMC University of Technology in 1995 and 1999, respectively. Then he graduated $\mathrm{PhD}$ degree from Heriot-Watt University, Edinburgh, UK in 2009. Now, he is a senior lecturer at Department of Electrical and Electronics Engineering, Ton Duc Thang university, Ho Chi Minh city, Vietnam. His research interests include Robotics, Computer Vision, Optimal and Intelligent Control.

Thang Trung Nguyen, he received his B.Eng. and M.Eng degrees in Electrical Engineering from University of Technical education Ho Chi Minh City, Ho Chi Minh city, Vietnam in 2008 and 2010, respectively. Now, he is teaching at department of electrical and electronics engineering, Ton Duc Thang university, Ho Chi Minh city, Vietnam. His research interests include optimization of power system, power system operation and control and Renewable Energy.

Dieu Ngoc Vo, he received B.Eng. and M.Eng. degree in electrical engineering from HCM City University of Technology, Vietnam, in 1995 and 2000, respectively and his D.Eng. degree in energy from Asian Institute of Technology (AIT), Pathumthani, Thailand in 2007. $\mathrm{He}$ is now teaching at Faculty of Electrical and Electronic Engineering, HCM City University of Technology, Vietnam. His interests are applications of $\mathrm{AI}$ in power system optimization, power system operation and control, power system analysis, and power systems under deregulation. 